\title{
Hardware Design of Noble MAC Protocol for Vehicle System
}

\author{
Yi-Nan $\mathrm{Xu}$ (Corresponding author) \& Ren-Zhuang Fu \\ Department of Electronics and Communication, Yanbian University \\ 977 Gongyuan Road, Yanji City, Jilin Province 133002, China \\ Tel: 86-433-273-3478Ｅ-mail: ynxu@ybu.edu.cn, 2074020308@ybu.edu.cn \\ $\mathrm{Yi}-\mathrm{Hu} \mathrm{Xu}$ \\ Division of Electronics and Information Engineering, Chonbuk National University \\ 664-14 Ga Deokjin Dong, Jeonju City, Jeonbuk 561-756, South Korea \\ Tel: 82-63-270-2466 E-mail: yhxu@jbnu.ac.kr
}

Received: March 15, 2011

Accepted: April 6, 2011

doi:10.5539/mas.v5n3p33

\begin{abstract}
Vehicular ad hoc networks (VANETs) are being designed to improve traffic safety and efficiency. Then it communicates with each other using sensed available spare spectra. In a VANET network, a medium access control (MAC) layer configuration determines a common set of channels to facilitate communication among participating nodes. Because spare spectra change dynamically according to time and locations, available spare channels of the number and the identity of each node are changing any time in a multi-hop network consisting of VANET nodes. In this paper, we present a novel dynamic hop reservation multiple access protocol based on the original one. Moreover, the MAC system is designed using Verilog HDL and implemented using ALTERA Excalibur ARM EPXA4F672C3 (FPGA). It can access and quit channels efficiently without any common channel.
\end{abstract}

Keywords: VANET, Medium access control, Hop reservation, FPGA

\section{Introduction}

Vehicle-to-Vehicle and Vehicle-to-Infrastructure communications have become. The IEEE $802.11 \mathrm{p}$ task group is working on the dedicated short-range communication standard to support wireless communications for vehicles and roadside infrastructures. Vehicle manufactures and telecommunication industries are gearing up to equip each car with onboard units that allow vehicles to communicate with each other, as well as to supply roadside units. VANET applications include active security, intelligent transportation, environmental protection and information service (Car-to-Car Communication Consortium)(H. Menouar, M. Lenardi, F. Filali. 1999)( FCC Spectrum Policy Task Force, 2005).

The key points of VANET are cognitive capabilities and adaptabilities(S. Yousefi, M. S. Mousavi, and M. Fathy. 2006). They enable communicating nodes to sense radio environment. Then system adapts itself to capture the frequency for keeping utilization and reliability. These tasks are finished with the processes of spectra sensing, spectra analysis, and spectra decision (A. Mishra. 2006). In the primary band, also known as spectrum holes (P. Papadimitratos, S. Sankaranarayanan and A. Mishra. 2005), spare spectra are identified firstly by system. Then, the system ensures secondary user to select the primary band. At a certain geographic location, there is more than one available primary band for the secondary users. These different primary bands have different traffic rules because of different applications of primary users. For example, they may be maximum power constraints or accessible bandwidth within the primary band, etc. Different using methods cause different maximum data rates available for secondary users. Considering such limitations, channel selection strategies are important for maximizing uses of primary bands.

In this paper, we illustrate hardware, a constrained media access control (MAC) protocol which explores spectrum opportunity. In order to design and implement hardware MAC system, the system is designed using Verilog HDL. In addition, the MAC system is implemented using ALTERA Excalibur ARM EPXA4F672C3. 
The rest of this paper is organized as follows. In section 2, presents the related previous work. Section 3 presents the detail MAC protocol design for VANET network. Section 4 presents the design of MAC protocol using Verilog HDL, and its implementation of FPGA. Finally, brief conclusions are given in section 5 .

\section{Review of Previous Work}

Each An Though design of MAC protocol for VANET is immature yet, there are several MAC protocols being developed with more flexible and efficient use of spectrum resource being built on top of the VANET. Several results will be mentioned below.

For reusing spectrum allocated to TV broadcast service, the IEEE 802.22 working group is in the process of standardizing a MAC layer based on VANET (IEEE 802.22 draft standard). The Channel Hopping Multiple Access (CHMA) (A. Tzamaloukas and J. Garcia-Luna-Aceves. 2000) uses a channel hopping approach method. That is, if nodes want to communicate with each other, they follow the same schedule. For example, if two nodes are able to exchange control information, they will stay on that channel to complete the data transfer. The Hop Reservation Multiple Access (HRMA) protocol (Z. Tang and J. J. Garcia-Luna-Aceves. 1999) is a multi channel MAC scheme for slow frequency hopping spread spectrum wireless ad hoc networks where all nodes hop according to a pre-defined hopping pattern. In this method, whenever a node has a data packet to send, the system exchanges RTS and CTS packets with the intended receiver, and both of them remain in the same hop for the entire data transmission. When multiple transceivers were in one place, the design of multi channel MAC protocols is significantly simplified. The multi channel MAC protocol proposed in the reference (A. Nasipuri and S. Das. 1999) assumes that each node has as many transceivers as there are channels. Here, nodes able to listen all these channels simultaneously. As the Dynamic Channel Assignment (DCA) algorithm proposed in the reference (S.-L. Wu, C.-Y. Lin, Y.-C. Tseng and J.-P. Sheu. 2000), control messages (RTS and CTS) are exchanged over a control channel. And data transfer takes place over a number of data channels. The main topic is the dedicated radio at the control channel and the problem of control channel.

\section{Hardware-Constrained VANET MAC Protocol}

In this section, we present the newly designed hardware constrained VANET MAC protocol.

\subsection{Node Characteristics}

We have an assumption that there are $\mathrm{N}$ of frequency channels as shown in Fig. 1. This assumption comes from consideration of military or relief operations where soldiers in a platoon or firemen assigned for a relief task is prioritized. In Fig. 1, every node is unique and it was pre-assigned. Nodes are assumed to be equipped GPS to enable physical location awareness and time synchronization among nodes (A. S. Brown, 2004).

\subsection{Frame Format}

The proposed VANET frame is consisted with two segments. They are header segment and payload segment as shown in Fig. 2 (FlexRay Communications System). The header segment bits define the basic feature of the frame, and payload segment ( 0 - 254 bytes) contains the main data.

In header segment, the Channel availability (1 bit) indicates the state of data transferring channel. Data showing the high bit of channel availability mean that this channel is available, or data showing the low bit of channel availability mean that this channel is not available.

The null frame (1 bit) indicates whether the frame is available or not. If the null frame shows high bit, it means that this frame is available. If the null frame shows low bit, it means that this frame is not available.

Hop reservation ( 7 bits) is common hopping sequence for the entire network and requires half duplex frequency hopping radios with no carrier sensing to operate.

Frame ID (11 bits) is designed as a slot position. The frame ID indicates the slot in which the frame should be transmitted. A frame ID is used no more than one time on each channel during one communication cycle. Each frame has a unique assigned frame ID corresponding with a unique slot. The frame ID ranges from 1 to 2047 (00000000001 to 11111111111), and the frame ID 0 is an invalid frame ID.

Data length ( 7 bits) is used to indicate the size of the payload segment. The payload segment size is encoded in this field by setting it to the number of payload data bytes divided by two (data length $\times 2$ = number of data bytes).

Adjacency environment (2bits) indicates the state of the channel that neigh boring channel is being used or not. First bit and last bit indicate the present or not of the higher rank channel and the lower rank channel, respectively. High bit and low bit mean the states of a working and a rest, respectively. 
GPS (15 bits) is corresponding to the GPS location of node position parameters, such as latitude (7 bits), latitude hemisphere ( 7 bits), longitude ( 7 bits), longitude hemisphere ( 7 bits) and height ( 7 bits).

The payload segment contains 0 to 254 bytes of data. The payload length contains the number of two-byte words ( 0 to 127 two-byte words). The bytes of the payload segment are identified numerically, starting at 0 for the first byte after the header segment and increasing by one with each subsequent byte. The individual bytes are referred as 'Data 0 ', 'Data 1', etc... 'Data 0 ' is the first byte of the payload segment, and 'Data N' is the last byte of the payload segment.

\subsection{State Diagram}

Fig. 3 shows the state diagram for proposed MAC. If one node wants to transmit, the system is set on a standby mode and the first communication cycle will be performed preferentially when the start message has been input from the host. Consequently, the state mode will be transferred from a standby mode to a wait for the cycle start mode. Then, frames corresponding with each slot will be transferred from a wait for the cycle start mode to a wait for the slot boundary mode. While each slot is decided in this way, according to the order of frame structures in Fig. 2, the frame will decide the message of channel availability, null frame, hop reservation, frame ID, data length, adjacency environment and GPS. Finally, the payload segment data is constructed. As an explanation above, each frame is constructed at its unique slot. The formed frame above will be transferred to a wait for the slot boundary state mode to construct a next frame according to the next slot.

\subsection{Frame Encoding}

In order to design the node encoding process of MAC system as an efficient and fault tolerant protocol. Fig. 4 shows the frame encoding process. The Transmission Start Sequence (TSS) is used to initiate proper connection setup through the network. A transmitting node generates a TSS that is consisted of a continuous 6 low bits. The Frame Start Sequence (FSS) is used to compensate for a possible quantization error in the first byte start after the TSS transferring signal. The FSS is consisted of one high bit. The Byte Start Sequence (BSS) is used to provide timing information of a bit stream to the receiving devices. Then, the BSS will be consisted of one high bit followed by one low bit. Each byte of frame data will be sent on the channel following BSS immediately. The Frame End Sequence (FES) is used to mark the end of the last byte in a frame. The FES is consisted of one low bit followed by one high bit. When transferring data, TSS, FSS, BSS, and FES appear periodically in a frame. If the system performs a frame encoding in this way, when the system is designed using hardware, the probability of data transmission will be improved, as well as, transmission errors will be decreased efficiently.

Figure 2 shows an example of a low-resolution image which would not be acceptable, whereas Figure 3 shows an example of an image with adequate resolution. Check that the resolution is adequate to reveal the important detail in the figure.

\section{Hardware Implementation}

The proposed MAC protocols can be realized in Finite State Machine (FSM) diagram form. FSM is a communication method to realize large and complicated system easily using software or hardware (Laurent Doldi, 2003). In this work, FSM method was used for easy implementation of MAC system.

For hardware implementation, MAC system was designed using Verilog HDL. And the designed system was simulated using Quartus II. Fig. 5 shows the timing simulation result of a transmit frame and event signals in channel A after transmission frame (T_Frame) being transmitted from the transmission node. The TxD signal is serial transmited data in Slot_No slot. Consequently, essential signals conforming a frame is output, such as channel availability (C_Ava), null frame (N_Frame), hop reservation (H_RSV), frame ID (F_ID), data length (D_Leg), adjacency environment (A_Env) end GPS (GPS).

To verify the designed MAC system, the MAC system is applied to FPGA. Fig. 6 shows the FPGA implementation for proposed MAC system using ALTERA Excalibur ARM EPXA4F672C3.

\section{Conclusions}

The hardware constraints VANET used by secondary users including sensing constraints and transmission constrains. In this paper, we have proposed a hardware constrained MAC protocol for VANET network, and presented a state diagram for global network topology and physical location of nodes in the network. In order to make the MAC system being designed more fault tolerant protocol, we mention the frame encoding process. The entire system is implemented using ALTERA Excalibur ARM EPXA4F672C3. It demonstrates that the designed MAC system operates successfully. 


\section{References}

A. Mishra. (2006). A Multi-channel MAC for Opportunistic Spectrum Sharing in Cognitive Networks. Proc. MILCOM 2006, October 2006, doi: 10.1109/MILCOM.2006.302013.

A. Nasipuri and S. Das. (1999). A Multichannel CSMA MAC Protocol for Multihop Wireless Networks. IEEE WCNC, 1999, doi: 10.1109/WCNC.1999.796968.

A. Tzamaloukas and J. Garcia-Luna-Aceves. (2000). Channel-hopping multiple access. IEEE ICC, June 2000, doi: 10.1109/ICC.2000.853348.

Car-to-Car Communication Consortium, http://www.car-to-car.org.

FCC Spectrum Policy Task Force. Report of the spectrum efficiency working group. Federal Communications Commision, USA, July 2005.

FlexRay Communications System - Protocol Specification, v2.1 Revision A, FlexRay Consortium, Dec. 2005.

IEEE 802.22 draft standard. IEEE P802.22 $22^{\mathrm{TM}} / \mathrm{D} 0.1$ Draft Standard for Wireless Regional Area Networks. http://www.ieee802.org/22/, doc. No.22-06-0068-00-0000, May 2006.

Klemp, O. (2010). Performance considerations for automotive antenna equipment in vehicle-to-vehicle communications. EMTS-2010, pp. 934-937, 2010, doi: 10.1109/URSI-EMTS.2010.5637361.

Laurent Doldi. (2003). Validation of Communications Systems with SDL. John Wiley, 2003.

P. Papadimitratos, S. Sankaranarayanan and A. Mishra. (2005). A bandwidth sharing approach to improve licensed spectrum utilization. Proc. First IEEE Symposium on New Frontiers in Dynamic Spectrum Access Networks, November 2005, doi: 10.1109/MCOM.2005.1561918.

S.-L. Wu, C.-Y. Lin, Y.-C. Tseng and J.-P. Sheu. (2000). A New Multi Channel MAC Protocol with On-Demand Channel Assignment for Multi Hop Mobile Ad Hoc Networks. I-SPAN, 2000, doi: 10.1109/ISPAN.2000.900290.

Yang WenQiang and Zhang JunCai. (2010). Research on the integrity monitoring of GPS Vehicle System. 2010 International Conference on E-Health Networking, Digital Ecosystems and Technologies, pp. 196-199, April 2010, doi: 10.1109/EDT.2010.5496396.

Yue Liu, Jun Bi and Ju Yang S. (2009). Research on Vehicular Ad Hoc Networks. Control and Decision Conference-2009, pp. 4030-4035, 2006, doi: 10.1109/CCDC.2009.5192343.

Z. Tang and J. J. Garcia-Luna-Aceves. (1999). Hop-Reservation Multiple Access (HRMA) for Ad Hoc Network. IEEE Infocom, 1999, doi: 10.1109/INFCOM.1999.749268.

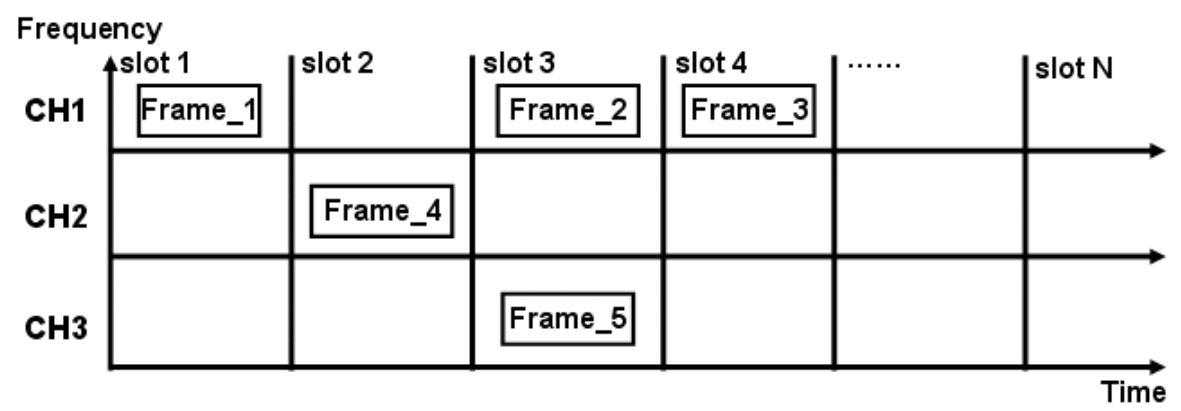

Figure 1. Operation cycle of the network 


\begin{tabular}{|c|c|c|c|c|c|c|c|c|c|c|c|}
\hline $\begin{array}{c}\text { Channel } \\
\text { Availability }\end{array}$ & $\begin{array}{l}\text { Null } \\
\text { Frame }\end{array}$ & $\begin{array}{c}\text { Hop } \\
\text { Reservation }\end{array}$ & $\begin{array}{c}\text { Frame } \\
\text { ID }\end{array}$ & \begin{tabular}{|c|} 
Data \\
Length
\end{tabular} & $\begin{array}{l}\text { Adjacency } \\
\text { Environment }\end{array}$ & GPS & Data 0 & Data 1 & Data 2 & $\cdots$ & Data N \\
\hline 1 bit & $1 \mathrm{bit}$ & 7 bits & 11 bits & 7 bits & 2 bits & 35 bit\$ & \multicolumn{5}{|c|}{0 ... 254 bytes } \\
\hline \multicolumn{7}{|c|}{ Header Segment } & \multicolumn{5}{|c|}{ Payload Segment } \\
\hline
\end{tabular}

Figure 2. VANET frame format

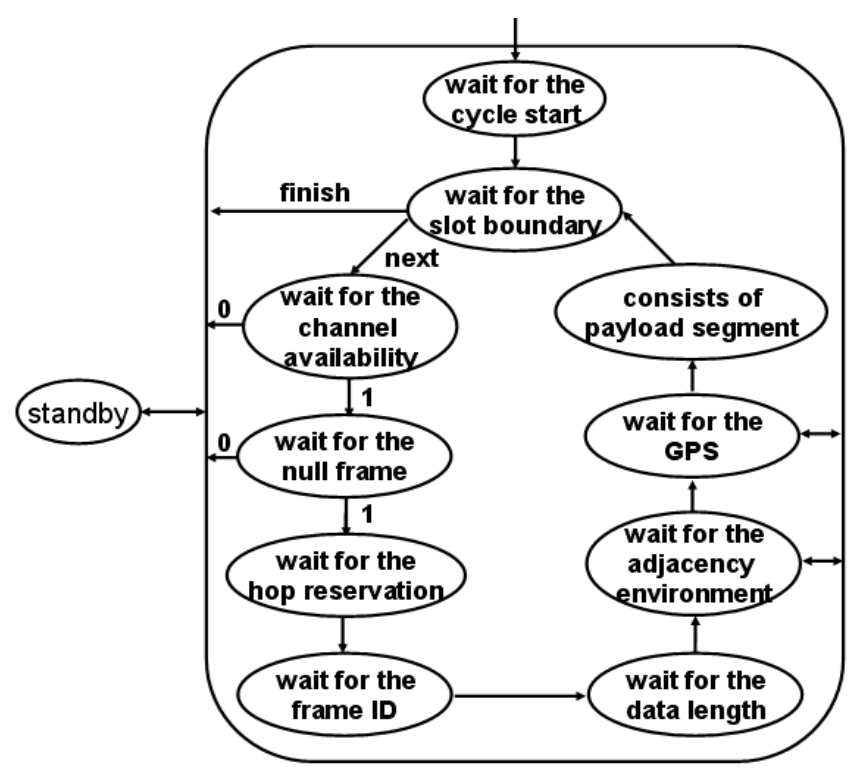

Figure 3. VANET frame format

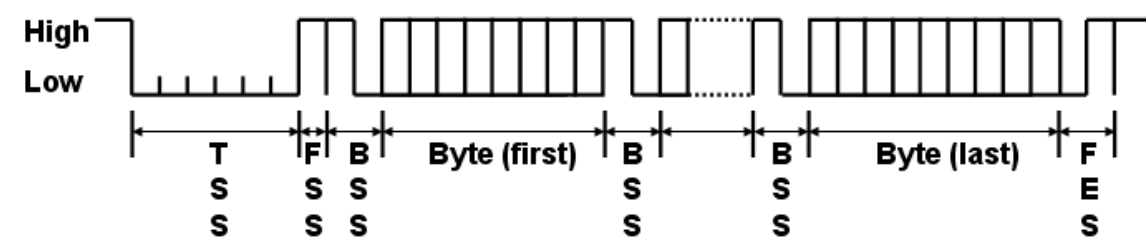

Figure 4. Hierarchical structure of FlexRay system 


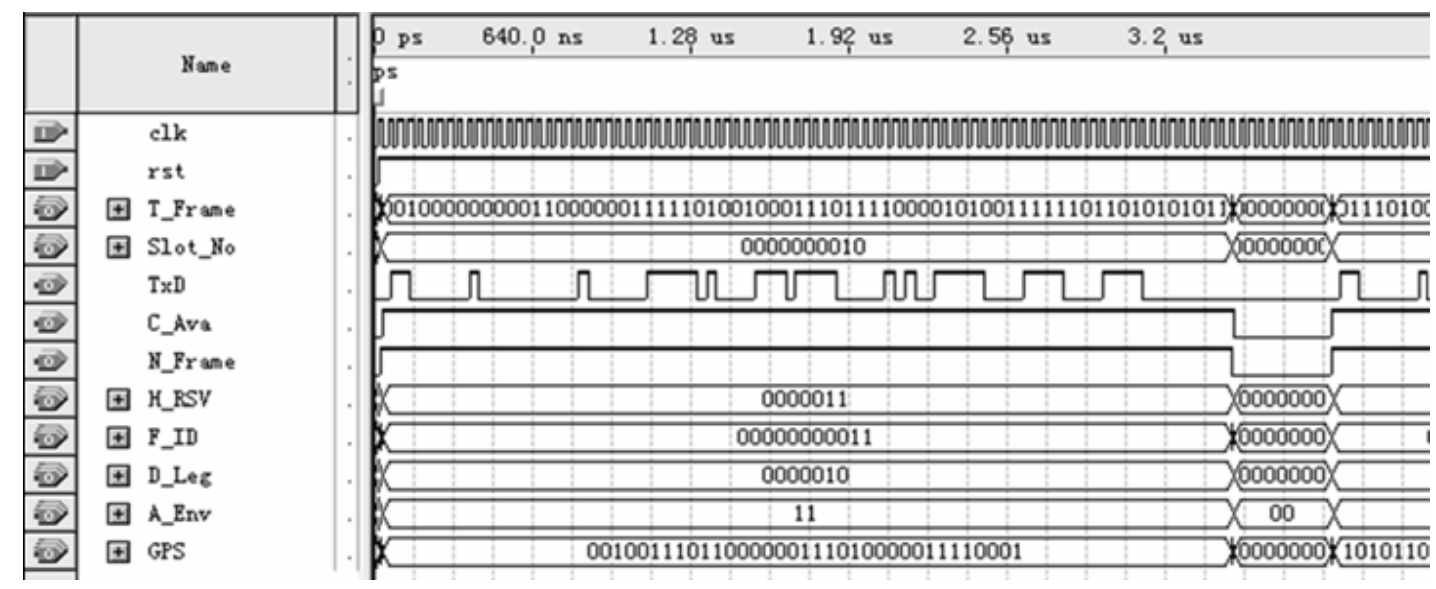

Figure 5. Timing simulation result of transmit frames

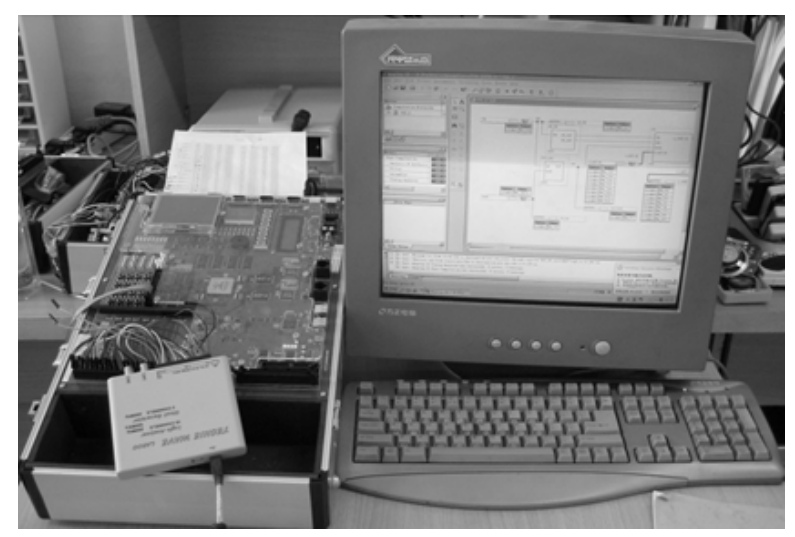

Figure 6. Real test environment of VANET MAC system 